\title{
Chlorpyrifos Induced Region Specific Vulnerability in Rat CNS and Modulation by Age and Cold Stress: An Interactive Study
}

\author{
P. Mahaboob Basha - Annappa Poojary
}

Accepted: 28 October 2010/Published online: 13 November 2010

(C) Springer Science+Business Media, LLC 2010

\begin{abstract}
Chlorpyrifos (CPF), an organophosphorus insecticide is known to cause ill health in non-target animals by inducing oxidative stress. In this study influence of cold stress $\left(15^{\circ} \mathrm{C}\right.$ and $\left.20^{\circ} \mathrm{C}\right)$ and age as modulating factors on $\mathrm{CPF}$ induced oxidative stress was addressed to assess age-related differences and vulnerability in central nervous system of rats. The results indicated an interaction with age and cold exposure resulting in marked decreased activity levels of SOD $(P<0.05)$, CAT $(P<0.05)$, GPx $(P<0.05)$, GST $(P<0.05)$ followed by increased MDA $(P<0.05)$ and decreased GSH levels $(P<0.05)$. The ANOVA and Post-hoc analysis showed that antioxidant enzymes decreased significantly $(P<0.05)$ on CPF exposure. Moreover synergistic action of $\mathrm{CPF}$ and cold stress at $15^{\circ} \mathrm{C}$ caused higher inhibition on comparison with CPF and cold stress alone and together at $20^{\circ} \mathrm{C}$ indicating the extent of peroxidative damage in discrete regions of CNS. Further this study showed young individuals to be more sensitive than adults.
\end{abstract}

Keywords Chlorpyrifos - Cold stress - Oxidative stress · Regional changes · Interactive effects

\section{Introduction}

Organophosphorous (OP) pesticide poisoning is often witnessed to be due to direct or accidental exposure and its entry into biological system of human or animal cause alterations

P. M. Basha $(\bowtie) \cdot$ A. Poojary

Department of Zoology, Bangalore University,

Bangalore 560 056, India

e-mail: pmbashabub@ rediffmail.com in many cellular enzymes including acetylcholinesterase and also affect various metabolic pathways. Chlorpyrifos $(\mathrm{O}$, $\mathrm{O}^{1}$-diethyl O-3, 5, 6-trichloro-2-pyridinyl phosphorothioate, $\mathrm{CPF}$ ) is the most widely used chlorinated OP insecticide and acaricide all over the world due to its moderate mammalian toxicity and greater stability and persistence [1]. Although the minimal risk level for oral exposure in humans is $0.003 \mathrm{mg} / \mathrm{kg}$, its presence in food substances has been reported to be in the range of $0.09-0.2 \mathrm{mg} / \mathrm{kg}$ [2]. The free radicals are believed to be responsible for lipid peroxidation which is a destructive, self-perpetuating chain reaction of oxidative destruction of lipids leading to the production of malondialdehyde, one of the biomarkers of oxidative stress. The harmful effects of CPF may occur as a result of its conversion to free radicals or via the formation of superoxide as a byproduct of its metabolism that cause disturbances in cell integrity leading to enhanced oxidative cell damage/ death $[2,3]$.

Earlier studies indicated cold exposure enhances the quantum of oxidative stress by increasing the pro-oxidants while depleting the antioxidant capacities [4, 5]. Further, it is also indicated that cold stress alters brain acetylcholinesterase levels when exposed to OP pesticides [6]. The limited data available suggest some differential effects on developing as well as maturing animals on exposure to CPF [7]. However, there is no report available in the literature that describes the interactive effects of acute CPF exposure and cold stress in discrete brain regions of different age group animals particularly neonatal, juvenile and young adults. The co-existence of OP toxicity and oxidative stress among various cell types tend to cause multitude of mechanisms that may either act simultaneously or consecutively. The extent to which the OP pesticide exposure impedes the functional ability of animal, especially on nervous system of different age group is not known. 
Keeping in view the lacuna in the literature and the role of cold stress in accelerating quantum of toxicity, the present study was undertaken to assess CPF induced region specific vulnerability to oxidative stress indices and its modulation by age and cold stress in rat central nervous system (CNS).

\section{Experimental Procedure}

\section{Chemicals}

Chlorpyrifos [(CAS: 2921-88-2), 20\% emulsified concentrate (EC), commercial grade] obtained from Lupin Agrochemicals Pvt Ltd, Gujarat (India). All other analytical grade chemicals were procured from BDH and SigmaAldrich.

\section{Animals}

Albino rats, wistar strain (Rattus norvegicus albinus) of different age groups viz., neonatal (7-day old), juvenile (21-day old) and young adult (90-day old) were procured from Sri Raghavendra enterprises, Bangalore and acclimatized to laboratory conditions $(12 \mathrm{~h}$ dark/light cycle at $28 \pm 2{ }^{\circ} \mathrm{C}$ ) for 1 week prior to commencement of the experiment. They were maintained on standard rodent pellet and tap water ad libitum; in accordance with the guidelines of National Institute of Nutrition, ICMR, Hyderabad and experimental protocol was approved by the Institutional animal ethical committee, Bangalore University, Bangalore.

\section{Design}

Rats were divided into six groups: Group I-control animals; Group II-exposed to CPF; Group III-exposed to cold stress at $15^{\circ} \mathrm{C}$; Group IV-exposed to cold stress at $20^{\circ} \mathrm{C}$; Group V-exposed to $\mathrm{CPF}$ plus cold stress at $15^{\circ} \mathrm{C}$; Group VI-exposed to CPF plus cold stress at $20^{\circ} \mathrm{C}$. The number of animals in each group was six. The group II, V and VI animals were treated with sub lethal doses (1/3 of maximum tolerated dose (MTD)) of CPF (subcutaneously) dissolved in dimethyl sulphoxide (DMSO) and rest of the animal groups were injected with plain DMSO. The MTD of CPF in rats is 45,127 and $279 \mathrm{mg}$ per $\mathrm{kg}$ body weight for neonatal, juvenile and young adult age groups respectively $[8,9]$. The cold stress group rats were housed in an acute cold stress apparatus (Colton BOD incubator) with a built-in heater and cooler that could be controlled by selftimer. Rats were sacrificed by cervical dislocation $48 \mathrm{~h}$ after respective treatments and discrete regions of CNS like cerebral cortex (CC), cerebellum (CB), medulla oblongata (MO) and spinal cord (SC) were quickly separated and washed in ice cold $0.9 \%$ saline. The tissue homogenates were made by using appropriate buffer and supernatant was stored at a temperature of $-20^{\circ} \mathrm{C}$ and used for oxidative stress parameter assays.

Biochemical Assays

\section{Lipid Peroxidation (LPO)}

Lipid peroxidation product was estimated by measurement of thiobarbituric acid reactive substances (TBARS) using the method of Niehaus and Samuelsson [10]. The pink chromogen produced by the reaction of thiobarbituric acid with malondialdehyde (MDA), a secondary product of lipid peroxidation was estimated at $535 \mathrm{~nm}$. Results are expressed as nmol of MDA formed/g wet weight of tissue.

\section{Superoxide Dismutase (SOD, EC 1.15.1.1)}

SOD activity was assayed by measuring the inhibition of epinephrine auto-oxidation as described by Misra and Fridovich [11]. The absorbance was recorded at $480 \mathrm{~nm}$ for $60 \mathrm{~s}$. Results are expressed as units/mg protein.

\section{Catalase (CAT, EC 1.11.1.6)}

CAT activity was measured as described by Aebi [12]. The rate constant of hydrogen peroxide $\left(\mathrm{H}_{2} \mathrm{O}_{2}\right)$ decomposition was monitored by measuring the decrease in absorbance at $240 \mathrm{~nm}$ for $60 \mathrm{~s}$. Results are expressed as nmol of $\mathrm{H}_{2} \mathrm{O}_{2}$ consumed/min/mg protein.

\section{Glutathione Peroxidase Activity (GPx, EC 1.11.1.9)}

GPx activity was estimated by measuring the oxidation of NADPH as described by Agergaard and Jensen [13] and change in absorbance was measured at $340 \mathrm{~nm}$. Results are presented as $\mu \mathrm{mol}$ of NADPH oxidized $/ \mathrm{min} / \mathrm{mg}$ protein.

\section{Glutathione-S-transferase (GST, EC 2.5.1.18)}

GST activity was estimated by the method of Habig et al. [14] by following the increase in absorbance at $340 \mathrm{~nm}$ using 1-chloro-2, 4-dinitrobenze (CDNB) as substrate. The assay was conducted by monitoring the appearance of the conjugated complex of CDNB and GSH, viz., 2, 4-dinitro phenyl glutathione at $340 \mathrm{~nm}$. Results are presented as nmol of 2, 4-dinitro phenyl glutathione formed $/ \mathrm{min} / \mathrm{mg}$ protein.

\section{Reduced Glutathione (GSH)}

Reduced glutathione content was determined using 5, $5^{1}$-dithio-bis (2-nitrobenzoic acid) (DTNB) as fluorescent 
reagent according to the method of Ellman [15]. GSH levels were monitored at $412 \mathrm{~nm}$. Results are expressed as $\mathrm{mg}$ of GSH/g wet weight of tissue.

\section{Protein Assay}

Protein content was estimated by the method of Lowry et al. [16], using bovine serum albumen (BSA) as a standard.

\section{Statistical Analysis}

The results are expressed as mean \pm standard deviation (SD) of six observations $(n=6)$ in each group. Differences between treatment groups of same age group were assessed by one-way analysis of variance (ANOVA) using the SPSS (version 15.0) software package for windows version 15.0. Post hoc testing was performed for inter-group comparisons using Bonferroni test at probability $(P)$ value $<0.05$ level of significance. To assess the interactions, three-way ANOVA was carried out and tested by Duncan's test for multiple comparisons to define the nature of the effect.

\section{Results}

Regional changes as a consequence of CPF toxicity and cold stress in different age group of animals are presented (Figs. 1, 2, 3, 4).

\section{Cerebral Cortex}

The effect of CPF exposure and cold interaction in different age group of rats in cerebral cortex is shown in Fig. 1. The three-way ANOVA indicated an interaction
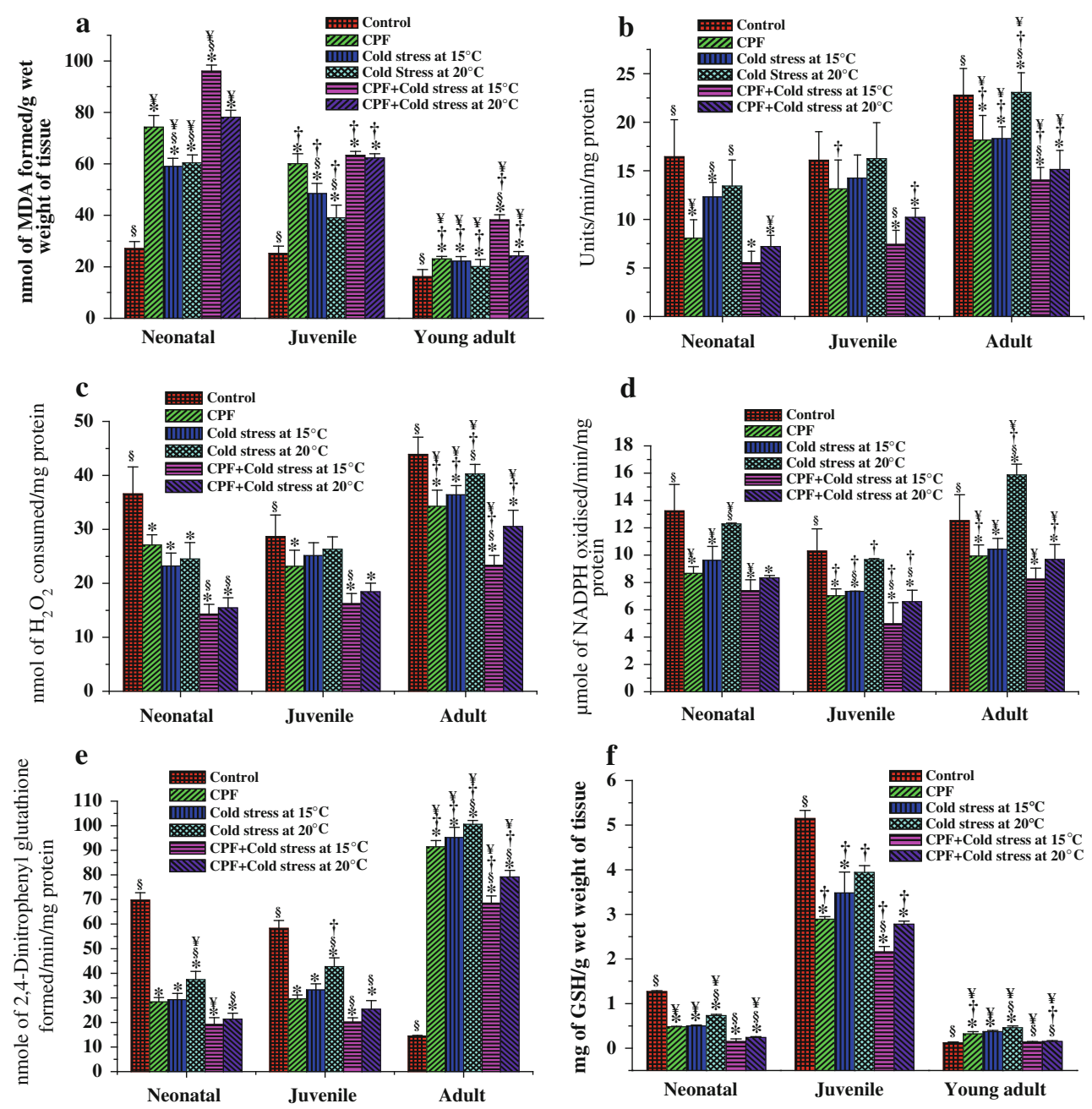

Fig. 1 Interactive effects of CPF and cold stress on oxidative stress indices in the cerebral cortex of different age group rats: a LPO level; b SOD activity; c CAT activity; d GPx activity; e GST activity; f GSH content. Results are presented as mean $\pm \mathrm{SD}, n=6$.

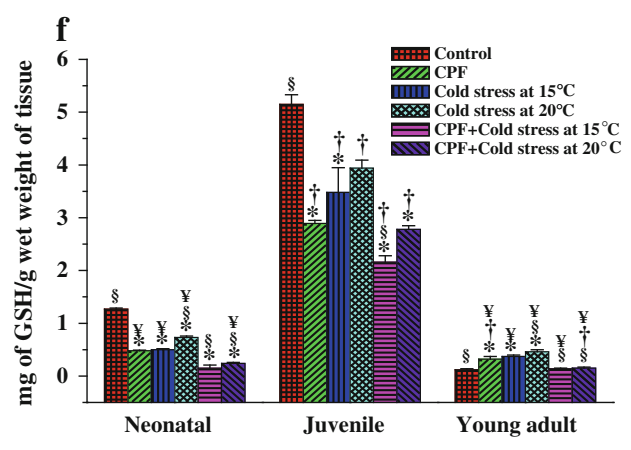

Significantly different from control: $* P<0.05$; significantly different from CPF: ${ }^{\&} P<0.05$; significantly different from neonatal in corresponding treatment groups: ${ }^{\dagger} P<0.05$; significantly different from juvenile in corresponding treatment groups: ${ }^{¥} P<0.05$ 
Fig. 2 Interactive effects of $\mathrm{CPF}$ and cold stress on oxidative stress indices in the cerebellum of different age group rats: g LPO level; $\mathbf{h}$ SOD activity; i CAT activity; j GPx activity; k GST activity; l GSH content. Results are presented as mean $\pm \mathrm{SD}, n=6$.

Significantly different from control: $* P<0.05$;

significantly different from CPF: ${ }^{\&} P<0.05$; significantly different from neonatal in corresponding treatment groups: ${ }^{\dagger} P<0.05$; significantly different from juvenile in corresponding treatment groups: ${ }^{¥} P<0.05$
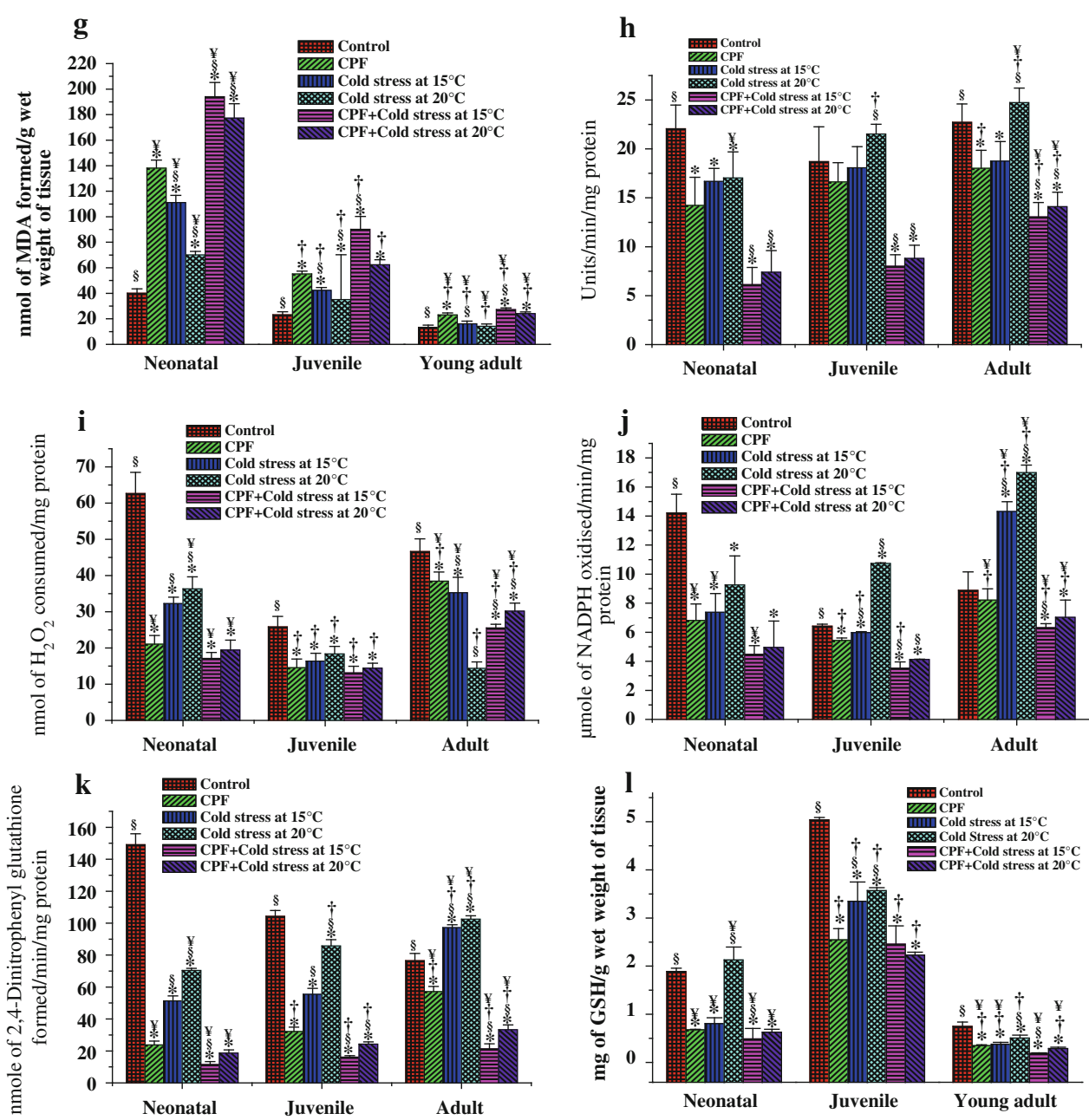

between age and cold exposure resulting in more decreased activity levels of SOD $(P<0.05)$, CAT $(P<0.05)$, GPx $(P<0.05)$ and GST $(P<0.05)$ followed by increased MDA levels $(P<0.05)$ and decreased GSH levels $(P<0.05)$. Post hoc analysis showed that SOD, CAT, GPx and GST activities were significantly decreased $(P<0.05)$ in $\mathrm{CPF}$ treated group. Moreover, interaction of $\mathrm{CPF}$ and cold exposure at $15^{\circ} \mathrm{C}$ presented higher inhibition with respect to antioxidant variables like SOD, CAT, GPx and GST activities as well as significantly increased levels of MDA and decreased level of GSH (all $P<0.05$ ) when compared to $\mathrm{CPF}$ and cold stress alone and together at $20^{\circ} \mathrm{C}$. Comparatively, neonatal animals presented higher sensitivity $(P<0.05)$ than juvenile and adult animals $(P<0.05)$, however, all the three age group animals showed lower levels of antioxidant enzymes $(P<0.05)$ when exposed to $\mathrm{CPF}$ and cold exposed together at $15^{\circ} \mathrm{C}$.

\section{Cerebellum}

The effect of CPF and cold exposure alone and together in cerebellum are shown in Fig. 2. The three-way ANOVA indicated a significant effect of CPF on the activities of SOD, CAT, GPx and GST to a greater extent when compared to other brain regions. Interactive effects were more pronounced in neonatal age groups exposed to $15^{\circ} \mathrm{C}$ over the other age groups.

\section{Medulla Oblongata}

The effect of CPF and cold exposure alone and together in medulla oblongata are shown in Fig. 3. The MDA levels were markedly $(P<0.05)$ higher in treatment groups as a consequence of $\mathrm{CPF}$ and cold stress indicating accelerated LPO in all the three age groups. The three-way ANOVA indicated a significant effect of CPF on the activities of SOD, CAT, and GPx $(P<0.05)$, and found interaction between age and cold on the activity of GST $(P<0.05)$. Neonatal animals presented higher level of MDA and GSH along with significantly decreased activity levels of SOD, CAT, GPx and GST $(P<0.05)$. Comparatively, this region appears to be more vulnerable in neonatal animals than juvenile and young adults. 

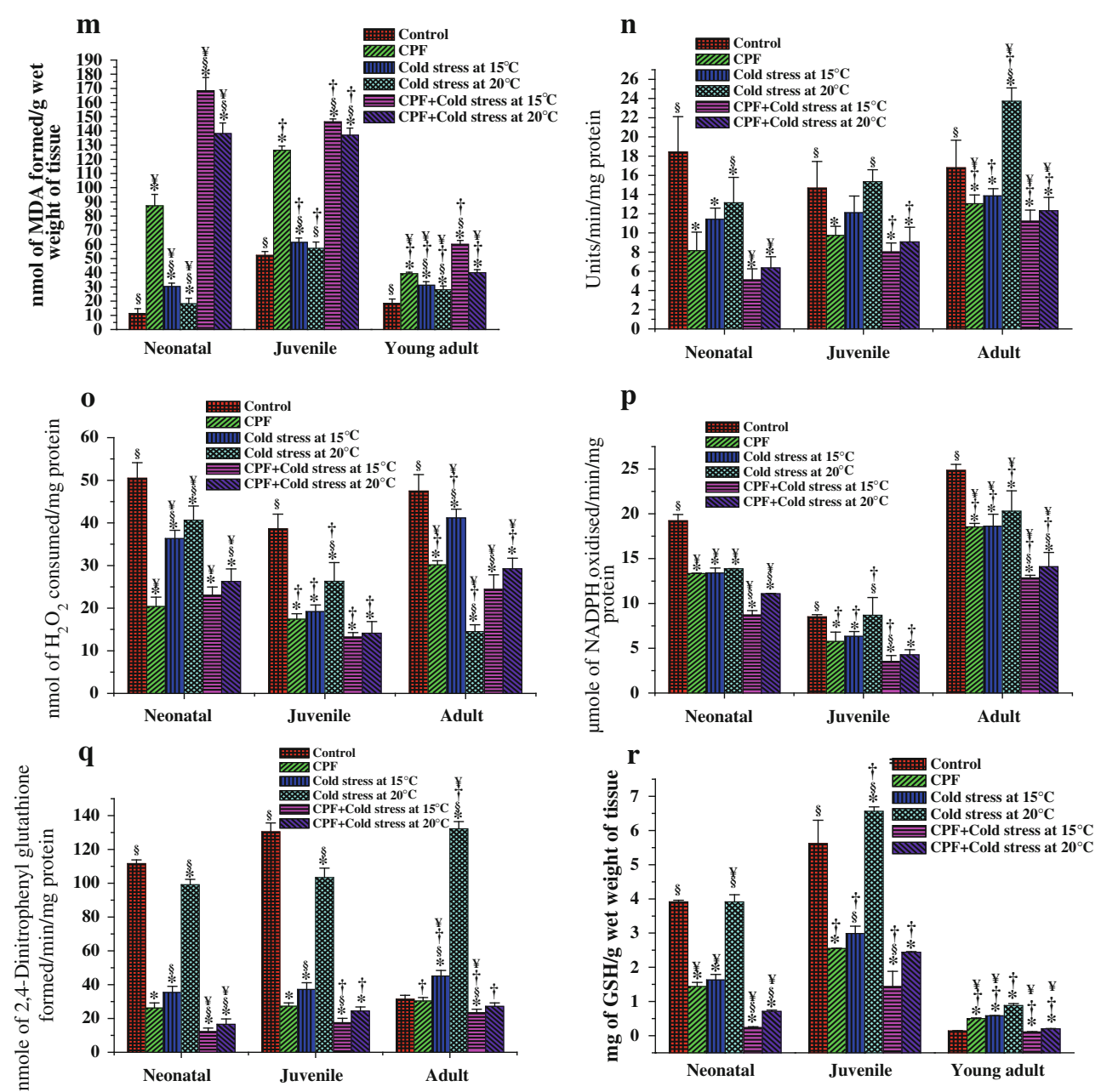

Fig. 3 Interactive effects of CPF and cold stress on oxidative stress indices in the medulla oblongata of different age group rats: $\mathbf{m}$ LPO level; $\mathbf{n}$ SOD activity; $\mathbf{o}$ CAT activity; $\mathbf{p}$ GPx activity; q GST activity; r GSH content. Results are presented as mean $\pm \mathrm{SD}, n=6$.

Significantly different from control: $* P<0.05$; significantly different from CPF: ${ }^{\&} P<0.05$; significantly different from neonatal in corresponding treatment groups: ${ }^{\dagger} P<0.05$; significantly different from juvenile in corresponding treatment groups: ${ }^{¥} P<0.05$

\section{Spinal Cord}

Interactive effects of $\mathrm{CPF}$ and cold stress on spinal cord are shown in Fig. 4. The three-way ANOVA indicated an interaction between age and cold exposure resulting in decreased activities of enzymes like SOD $(P<0.05)$, CAT $(P<0.05)$, GPx $(P<0.05)$ and GST $(P<0.05)$ along with increased MDA levels $(P<0.05)$ and decreased level of GSH in treatment groups of all the three age groups. Exposure to $15^{\circ} \mathrm{C}$ modified the CPF toxicity by accelerating the inhibition of antioxidant enzymes $(P<0.05)$.

\section{Discussion}

The oxidation of pesticides in vivo involves several mechanisms including the formation of reactive oxygen species

(ROS) which are highly damaging to biological tissues and their generation appears to be important to assess mode of action of a variety of pesticides. Generation of free radicals, lipid peroxidation (LPO) and altered antioxidant systems are considered to play a vital role in posing toxic effects of $\mathrm{CPF}$. Increased oxidative stress has also been directly linked to oxidation of cellular macromolecules which may cause injury to the CNS or induce a variety of adverse cellular responses. Interference of $\mathrm{CPF}$ with normal neurite development through a variety of mechanisms has been reported in the previous studies [17-20].

Examination of CNS vulnerability under the exposure conditions of cold stress and CPF toxicity and their interaction in different age groups (neonatal, juvenile and young adult rats) exhibited more pronounced alterations in the antioxidant levels along with MDA in neonatal rats followed by juveniles when compared to young adults. SOD, 
Fig. 4 Interactive effects of $\mathrm{CPF}$ and cold stress on oxidative stress indices in the spinal cord of different age group rats: $\mathbf{s}$ LPO level; $\mathbf{t}$ SOD activity; u CAT activity; v GPx activity; w GST activity; $\mathbf{x}$ GSH content. Results are presented as mean $\pm \mathrm{SD}, n=6$.

Significantly different from control: $* P<0.05$; significantly different from CPF: ${ }^{\&} P<0.05$; significantly different from neonatal in corresponding treatment groups: ${ }^{\dagger} P<0.05$; significantly different from juvenile in corresponding treatment groups: ${ }^{¥} P<0.05$
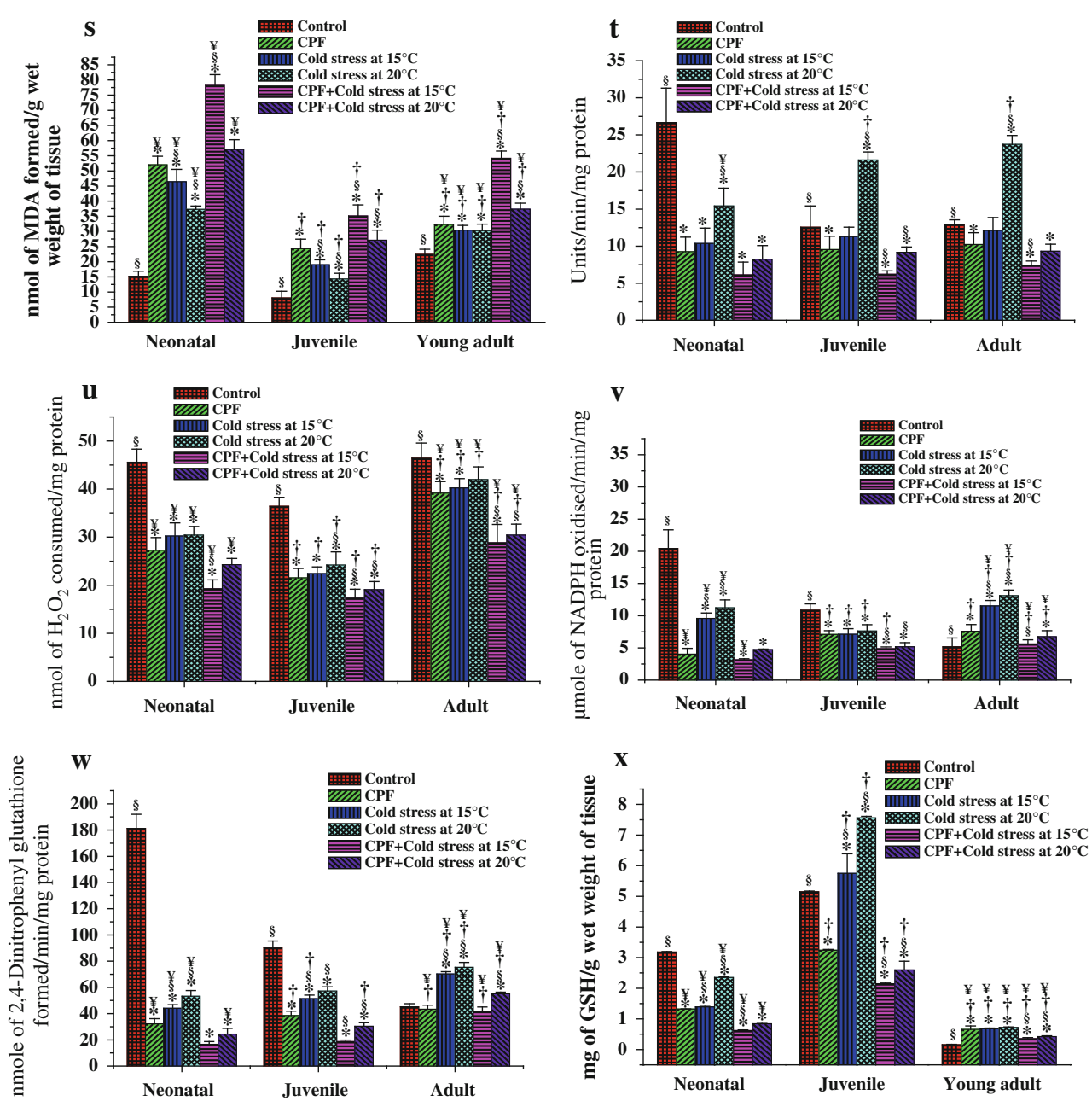

the first line of defense against the superoxide radical is found to decrease significantly with decreased age group. The decrease found in the activity of SOD on CPF exposure in the present study may reflect tissue injury due to accumulation of $\mathrm{O}_{2}^{\bullet}$ radicals. SOD being an incomplete antioxidant and its biological action is connected with CAT via $\mathrm{H}_{2} \mathrm{O}_{2}$. The decrease observed in CAT activity with decreased age group on CPF exposure indicates its reduced ability to protect neuronal cells against hydrogen peroxides. Further, both SOD and CAT would have failed to combat the ROS, as superoxide anion inhibits catalase action and the presence of hydrogen peroxide inhibits the action of dismutase [21].

GSH, a non-enzymatic potent cellular antioxidant acting as a redox buffer, is known to protect cellular constituents from oxidative damage by reacting directly with oxidants or by acting as substrate for GSH peroxidase (GPx) to scavenge peroxides [22]. Similarly GPx, a selenium containing enzyme catalyses the reduction of variety of hydrogen peroxides $\left(\mathrm{ROOH}\right.$ and $\mathrm{H}_{2} \mathrm{O}_{2}$ ) using glutathione as a substrate, thereby protecting mammalian cells against oxidative stress. A decrease observed in the activity levels of GPx with decreased age group on CPF exposure may render the tissue more susceptible to lipid peroxidative damage. Further a decrease found in the level of GSH in all three age groups studied more so in neonates and juveniles compared to young adults would suggest its inefficiency in protecting cellular constituents from oxidative damage or to scavenge the peroxides. Inspite of having sufficient reserves of glutathione system to protect the cell against the effects of ROS, possibly the cold stress might have suppressed the total glutathione level in young adult rats. Moreover, a decrease in the level of GSH may be accomplished by an increase in MDA level. Detoxifying enzyme GST catalyzes the conjugation of GSH with a compound having a reactive electrophilic carbon center, resulting in the formation of less toxic products for excretion. A low activity of GST as found in the present study may render the CNS more prone to lipid peroxidative damage. Earlier studies have indicated that the activities of enzymes associated with antioxidant defense are altered on insecticide exposure, both in vivo as well as in vitro 
[3, 23-25] corroborate our findings. From this study it is clear that neonatal and juvenile rats are at higher risk than the young adult because of relative deficiency of enzymatic and non- enzymatic antioxidants. There seems to be a maturation-dependant window of vulnerability to free radical attack.

In this study, we demonstrated that $\mathrm{CPF}$ intoxication induces oxidative stress in discrete regions of CNS, which is influenced by age and cold stress through the generation of free radicals and bring alterations in the cellular antioxidant defense system. A balance between the extent of metabolic activation and detoxification determines individual susceptibility to toxic actions of CPF which is most likely associated with individual and age-dependent sensitivity. The maturing animals viz., neonates and juveniles are more susceptible to acute toxic effects of CPF than young adults, primarily because of lower levels of detoxifying enzymes. The detoxification of CPF include binding to carboxylesterases and hydrolysis by arylesterases [26] and these mechanisms appear to have got altered differentially in different age group of rats since physiologic systems differ in different age group leading to homeostatic alterations, as a result the ability to respond adequately to toxic stress or insult varies. Young animals are unique because of the immature nervous system which responds differentially to the exposure of CPF with its kinetic ability (absorption, distribution, metabolism \& excretion). The greater neonatal sensitivity primarily has been attributed to the lack of complete metabolic competence [27]. Neonatal rats are more vulnerable because of active acquisition of iron for differentiation at the time of relative delay during the development of certain key antioxidant defenses in the brain. Further, excess free iron and deficient iron binding and metabolizing capacity are additional features favouring oxidative stress in younger rats than adults on CPF exposure. In excess, iron helps superoxide anion $\left(\mathrm{O}^{\bullet-}\right)$ and $\mathrm{H}_{2} \mathrm{O}_{2}$ to be converted into the extremely reactive hydroxyl radical $\left(\mathrm{OH}^{\bullet}\right)$ (Haber-Weiss reaction) that cause severe injury to membrane proteins and DNA [28]. ROS burden in various brain regions is due to altered neuronal plasticity, well known heterogeneity and metabolic compartmentalization of brain mitochondria [29]. The brain tissue being heterogeneous with a large number of different neuronal and non-neuronal cell types, different regional activities of antioxidant systems and variable metabolic rates lead to a region-specific accumulation of oxidative damage, and such differences would have increased the vulnerability of specific brain regions to age-dependent oxidative stress. Further, compartmentalization of CNS appears to be selectively vulnerable to oxidative cellular damage because of their differential oxidative metabolic ability and poor antioxidant defense, presence of susceptible proteins (high disulphide bonds), oxidizable lipids, rich iron and post mitotic cells [30]. A high rate of oxygen consumption coupled with low potential of brain to obviate oxidative stress might be the main triggering factor for the enhanced release of ROS during CPF exposure. Perhaps young rat brain as a whole appears to be susceptible to injury by free radicals; the cerebellum and medulla seem to be more prone to oxidative damage due to their higher oxygen consumption rate and the presence of iron which catalytically involved in the production of ROS.

Our results suggested that the pervasive influence of cold stress caused a significant increase in LPO and a marked decrease in antioxidants. The generalized decrease found in the levels of SOD, CAT, GPx, GST and GSH on acute cold stress exposure in rats indicate their highly reduced ability to scavenge ROS. Congruent with our findings, Kaushik and Kaur reported that cold stress could generate oxidative stress by altering the pro-oxidant-antioxidant balance of the free radical process in rat tissues [4]. It is assumed that cold stress stimulates the para-sympathoadrenal system causing inactivation of catecholamine biosynthetic enzymes [31] and elevating the free radical production in brain triggering catecholamine metabolism such as dopamine and nor adrenaline which in turn stimulates ROS over production [32]. Depletion in the activity of enzymatic and non-enzymatic antioxidants can be owed to an enhanced free radical production during cold stress. Moreover, physiological response to cold stress is by the activation of hypothalamic-pituitary-adrenal axis and subsequent release of corticosteron from the adrenal cortex into the blood stream [33] and the augmented endogenous corticosteron due to stress response [34] would have accelerated the generation of free radicals. In the present study an increase found in LPO was presumably associated with increased free radicals that would in turn inhibit the activities of SOD, CAT, GPx and GST. The observed reduction might attribute to the utilization of these antioxidants to alleviate free radical induced oxidative stress.

When CPF intoxicated rats were exposed to temperatures at $15^{\circ} \mathrm{C}$ and $20^{\circ} \mathrm{C}$, their interaction exacerbated, in resulting synergistic action that substantially modified the toxicity in discrete regions of CNS indicating severe effect of $\mathrm{CPF}$ and the quantum of synergistic interaction was most apparent at $15^{\circ} \mathrm{C}$. In line with our findings, it has been reported that concomitant exposure to $\mathrm{CPF}$ and cold stress exaggerates the neurotoxicity in animals by provoking the acetyl cholinesterase inhibition [6]. In true sense, the mode of action of $\mathrm{CPF}$ in cold temperate regions would have differential toxic actions than tropical regions indicating negative temperature coefficient ability (i.e., greater toxicity at lower temperature). Earlier studies suggested more toxic actions of certain insecticides at higher temperatures [35] while others show stronger lethal action at cold temperatures [36]. The decrease in environmental temperature 
may decrease the CPF degradation resulting in its longstanding effect. Perhaps stability, vaporization, penetration, activity and degradation of insecticides are all partly dependent on physical and biochemical processes that proceed at characteristic rates at different temperature [37].

Conclusively, it can be avowed that both CPF and cold stress cause oxidative stress variably and their interaction exert a different degree of influence on enzymatic and nonenzymatic antioxidants in different age group of rats, thereby exacerbates the oxidative damage to a greater extent. Further, the age also play a pivotal role in the modulation of cellular mechanisms of pesticide toxicity. Based on these findings it is tempting to speculate that environmental cold temperature may act as a synergistic factor to modify the quantum of CPF toxicity in modulating the cellular homeostasis.

Acknowledgments This study was supported by University Grant Commission (UGC), New Delhi, India under the Faculty Improvement Programme (FIP) for co-author.

\section{References}

1. Krishik V, Maser D (2006) Japanese beetle management in Minnesota. University of Minnesota US FO-07664

2. Alvarez AA, Ramirez-San JE, Canizales-Roman A (2008) Chlorpyrifos induces oxidative stress in rats. Toxicol Environ Chem 90:1019-1025

3. Zama D, Meraihi Z, Tebibel S, Benayssa W, Benayache F, Benayache S, Vlietinck AJ (2007) Chlorpyrifos-induced oxidative stress and tissue damage in the liver, kidney, brain and fetus in pregnant rats: the protective role of the butanolic extract of Paronychia argentea L. Indian J Pharmacol 39:145-150

4. Kaushik S, Kaur J (2003) Chronic cold exposure affects the antioxidant defense system in various rat tissues. Clin Chim Acta 333:69-77

5. Joanisse DR, Storey KB (1996) Oxidative stress and antioxidants in overwintering larvae of cold-hardy golden rod gall insects. J Exp Biol 199:1483-1491

6. Maquire CC, Williams BA (1996) Response of thermal stressed bobwhite to organophosphorus exposure. Rev Environ Health 11(3):101-117

7. Zheng Q, Olivier K, Won YK, Pope CN (2000) Comparative cholinergic neurotoxicity of oral chlorpyrifos exposures in preweanling and adult rats. Toxicol Sci 55:124-132

8. Pope CN, Chakraborti TK, Chapman ML, Farrar JD, Arthun D (1991) Comparison of in vivo cholinesterase inhibition in neonatal and adult rats by three organophosphorothioate insecticides. Toxicol 68:51-61

9. Zhang H, Liu J, Pope CN (2002) Age-related effects of chlorpyrifos on muscarnic receptor-mediated signaling in rat cortex. Arch Toxicol 75:676-684

10. Niehaus WG Jr, Samuelsson B (1968) Formation of malonaldehyde from phospholipid arachidonate during microsomal lipid peroxidation. Eur J Biochem 6:126-130

11. Misra HP, Fridovich I (1972) Role of superoxide radicals in the acute auto oxidation of epinephrine; a simple assay for SOD. J Biol Chem 247:70-75

12. Aebi H (1984) Catalase in vitro. Methods Enzymol 105:121-126
13. Agergaard N, Jensen PT (1982) Procedure for blood glutathione peroxidase determination in cattle and swine. Acta Vet Scand 23:515-527

14. Habig WH, Pabst MJ, Jakoby WB (1974) Glutathione S-transferases: the first enzymatic step in mercapturic acid formation. J Biol Chem 249:7130-7139

15. Ellman GL (1959) Tissue sulfhydryl groups. Arch Biochem Biophys 82:70-77

16. Lowry OH, Rosebrough NJ, Farr AL, Randal RJ (1951) Protein measurement with the Folin phenol reagent. J Biol Chem 193:265-275

17. Campbell CG, Seidler FJ, Slotkin TA (1997) Chlorpyrifos interferes with cell development in rat brain regions. Brain Res Bull 43:179-189

18. Garcia SJ, Seidler FJ, Slotkin TA (2005) Developmental neurotoxicity of chlorpyrifos: targeting glial cells. Environ Toxicol Pharmacol 19:455-465

19. Roy TS, Sharma V, Seidler FJ (2005) Quantitative morphological assessment reveals neuronal and glial deficits in hippocampus after a brief subtoxic exposure to chlorpyrifos in neonatal rats. Brain Res Dev Brain Res 155(1):71-80

20. Slotkin TA, Seidler FJ, Fumagalli F (2008) Targeting of neurotrophic factors, their receptors, and signaling pathways in the developmental neurotoxicity of organophosphates in vivo and in vitro. Brain Res Bull 76:424-438

21. Kono Y, Fridovich I (1982) Superoxide radical inhibits catalase. J Biol Chem 257:5751

22. Beutler E (1989) Nutritional and metabolic aspects of glutathione. Annu Rev Nutr 9:287-302

23. Fortunato JJ, Feier G, Vitali AM, Petronilho FC, Dal-Pizzol F, Quevedo J (2006) Malathion-induced oxidative stress in rat brain regions. Neurochem Res 31:671-678

24. Vidyasagar J, Karunakar N, Reddy MS, Rajnarayana K, Surender T, Krishna DR (2004) Oxidative stress and antioxidant status in acute organophosphorus insecticide poisoning. Indian J Pharmacol 36:76-79

25. Gultekin F, Delibas N, Yasar S, Kilinc I (2001) In vivo changes in antioxidant systems and protective role of melatonin and a combination of vitamin $\mathrm{C}$ and vitamin $\mathrm{E}$ on oxidative damage in erythrocytes induced by chlorpyrifos-ethyl in rats. Arch Toxicol 75:88-96

26. Karanth S, Pope CN (2000) Carboxylesterase and A-esterase activities during maturation and aging: relationship to the toxicity of chlorpyrifos and parathion in rats. Toxicol Sci 58:282-289

27. Benke GM, Murphy SD (1975) The influence of age on the toxicity and metabolism of methyl parathion and parathion in male and female rats. Toxicol Appl Pharmacol 31:254-269

28. Stadtman ER (1992) Protein oxidation and aging. Science 257:1220-1224

29. Villa RF, Gorini A, Zanada F, Benzi G (1986) Action of L-acetylcanitine on different cerebral mitochondria populations from hippocampus. Arch Int Pharmacodyn Ther 279:195-211

30. Floyd RA (1999) Antioxidants, oxidative stress, and degenerative neurological disorders. Proc Soc Exp Biol Med 222:236-245

31. Venarucci D, Venarucci V, Vallese A, Battila L, Casado A, De La Torre R, Lopez Fernandez ME (1999) Free radicals: important causes of pathologies refer to ageing. Panminerva Med 41:335-339

32. Carpagnano GE, Kharitonov SA, Resta O, Foschino-Barbaro MP, Gramiccioni E, Barnes PJ (2003) 8-Isoprostane, a marker of oxidative stress, is increased in exhaled breath condensate of patients with obstructive sleep apnea after night and is reduced by continuous positive airway pressure therapy. Chest 124:1386-1392

33. Owens MJ, Nemeroff CV (1991) Philosophy and Pharmacology of corticotrophin releasing factor. Pharmacol Rev 91:425-473

34. McInntosh LJ, Sapolsky RM (1996) Glucocorticoids increased the accumulation of reactive oxygen species and enhance 
adriamycin-induced toxicity in neuronal culture. Exp Neurol 141:201-206

35. Arthur FH, Dowdy AK (2003) Impact of high temperatures on efficacy of cyfluthrin and hydroprene applied to concrete to control Tribolium castaneum (Herbst). J Stored Prod Res 39:193-204
36. Horn DJ (1988) Ecological approach to pest management. Guilford, New York, p 285

37. Felsot AS (1989) Enhanced biodegradation of insecticides in soil: implications for agroecosystems. Ann Rev Entomol 34:453-476 